\title{
Use of Open Source Software for the Development of Web GIS for Accessibility to Tourist Attractions
}

\author{
Maria Teresa Françoso ${ }^{1}$, Diogenes Cortijo Costa ${ }^{1}$, Mariana Mello Valin ${ }^{2}$ and Rogério Rodrigues Amarante ${ }^{3}$ \\ 1. Department of Geotechnics and Transports, School of Civil Engineering, Architecture and Urban Design, University of Campinas, \\ São Paulo 13083-852, Brazil \\ 2. JBS S.A., Corporation, Lins 16402-161, Brazil \\ 3. Campinas Town Hall, Americana 13468-100, Brazil
}

\begin{abstract}
Based on the concepts of social inclusion, universal design and accessibility, this paper aims to describe the stages of the development and implementation of a Web GIS (geographic information system for the internet) based on OS (open source) software. The Web GIS developed is called "accessibility to Campinas tourist attractions”, which provides information about cultural and natural attractions in Campinas/SP-Brazil, especially for people with disabilities or reduced mobility that prevents access these sites via public and private transportation. The verification of the existence or lack of accessibility equipment at bus stops and parking areas close to tourist attractions relies on current legislation (NBR 9050/1994) that establishes the right to accessibility for disabled people to transportation, local culture and leisure. An application that facilitates this recognition with low operating costs and high performance was developed using the OS software Quantum GIS 1.0.2, Apache 2.2.4, Mapserver 5.0.2, I3GEO 4.0, PostgreSQL 8.3 and PostGIS 1.3.5. This software is able to integrate the management of tourist areas and the advertisement of tourist information regarding accessibility devices at sights recognized as tourist attractions by Campinas city hall.
\end{abstract}

Key words: Geographic information systems, Web GIS, transportation, tourism, accessibility, open source software.

\section{Nomenclature}

Acess_particular

Acess_publico

Acessibilidade local turístico

Acessibilidade transporte

particular

Acessibilidade transporte

public

Acessivel

Atrativo

Atrativo cultural

Atrativo natural

Distancia_ponto

Inacessíel

Parcialmente acessíel

Pontos

Tipo_acessibilidade

Vagas_estacionamento

\section{Introduction}

Some social groups that could enthusiastically participate in tourism, culture and leisure activities

Corresponding author: Maria Teresa Françoso, Ph.D., research field: transportation. E-mail: mteresa@fec.unicamp.br. offered by cities, which are unable because of a lack of facilities that meet their needs, and more importantly, information about the accessibility to these places [1]. Therefore, it is crucial to advertise information about tourism and leisure to people with reduced mobility or disabilities.

The growing development of computers and telecommunications has made it possible to create systems capable of storing and disseminating large amounts of information. Initially, GISs (geographic information systems) assumed this role, but access was restricted to users of a particular local area network (intranet). Soon after, Web GIS became popular due to the need to share spatial information with many users via the internet [2]. Web GIS is a hypertext-based service that allows users to browse information available on network computers that disseminate spatial data on the internet. The use of Web GIS has been growing significantly in recent 
years, which is reflected by the increase in the number of GIS workstations distributed across the World Wide Web [3]. In general, Web GIS features an easy and intuitive interface for the presentation of thematic maps, with some basic functionality for the manipulation and control of various types of content [4].

The ease of use by the non-specialized public combined with the power of cartographic communication are the major reasons for the popularity of these systems [4].

In this way, Web GIS applications open up new possibilities for the planning and management of economic activities such as tourism because they allow for the contribution to the greater socialization of information. Thus, tourism managers can find Web GIS to be a useful tool for the planning and analysis of environmental data or distribution of services. Thus, users can access detailed information about tours on the internet [5].

Because it contains spatial information, Web GIS also allows tourists to locate attractions of interest and check for accessibility equipment and the possibility of access through private or public transportation, making it possible to plan visits. This information must be provided simply and directly because most users are not usually map-reading experts [6].

The development of a GIS and a Web GIS can be expensive, often making it impractical for the tourism sector. However, OS (open source) software, such as Quantum GIS, I3Geo and PostgreSQL/PostGIS, is available to this sector. The advantages of these technologies range from a no-cost software license to the flexibility of adapting the program to meet actual needs without depending on closed software.

Therefore, this study aims to show that it is possible to implement a Web GIS dedicated to the management and provision of information regarding the cultural and natural tourist attractions of Campinas City using OS software. The goal is the production of thematic maps to identify the sights most likely to be accessed by private and public transportation to improve information about accessibility devices as a way to promote the social inclusion of people with physical disabilities or reduced mobility.

The purpose of this work is justified not only because the rights to leisure and transportation are guaranteed to all citizens but also because there is a lack of studies related to this theme, as well as to contribute to social inclusion by encouraging excluded groups to enjoy tourism, arts and leisure. It is known that the special characteristics of certain groups can highlight the importance of a space itself as a means for people to enjoy tourist sites in a way that is compatible with their physical differences or peculiarities.

\section{Web GIS for Accessibility to Tourist Attractions of Campinas/SP-Brazil}

Campinas/SP-Brazil was chosen for this study due to the interest of the Tourism Department of the Campinas City hall in expanding the city's accessible tourism and information about accessibility devices to tourist attractions. The existence of a digital cartographic database of the city led to this choice. After the analysis of available data, the survey was restricted to tourist attractions located in the urban area of Campinas, which were defined by the Municipal Tourism Secretary and are listed in Ref. [7].

The digital cartographic database of Campinas used in this study was provided by the "EMDEC (Empresa Municipal de Desenvolvimento de Campinas), Municipal Company of Campinas Development”, it features .dxf and .tab formats and uses the Hayford ellipsoid as a reference surface and the horizontal datum of Córrego Alegre and UTM (universal transverse Mercator) coordinate system. It should be noted that some layers of this database were in the geographic coordinate system and thus had to be converted to the UTM coordinate system.

It was also necessary to convert the layers to the 
shapefile format and later to sql script, to make them compatible with the Quantum GIS, I3GEO and PostgreSQL/PostGIS software. The OS software AutoCad DXF to Shapefile Converter, FWTools, DXF2PostGIS and shp2pgsl were used for the conversions.

The database was elaborated in the DBMS (Data Base Management System) PostgreSQL 8.3/PostGIS 1.3.5 with the console PgAdmin III 1.8. The entities and attributes are designed to be filled with data collected in the field and thus facilitate the preparation of thematic maps by executing spatial queries.

A total of 59 sights considered to be tourist attractions by the Department of Tourism of Campinas City were visited to collect data on accessibility. In January and February 2009, technical visits were made to these sights to identify the existence of reserved parking and equipment access at sights of touristic interest as a way of measuring accessibility to the sights by private transportation. The existence of suitable transportation routes near the attractions defined in this study was also verified as a way to measure the accessibility to the sight by public transport. Locational accessibility (distance between the bus stops and tourist attractions) and existing facilities at bus stops were also assessed, which included elements that facilitate user access such as ramps, paving, etc.. These elements were used to classify the accessibility to city bus stops. The survey also looked for the existence of accessible routes that permit smooth travel without barriers between the point of access and the final destination. All of these analyses were based on the premises of universal design and the NBR 9050/1994 [8].

The satellite receiver of the Garmin eTrex model GPS (global positioning system) was used in navigation mode without differential correction to obtain the coordinates of the geographical location of tourist attractions. In addition, the free version of the Brazilian software GPS TrackMaker [9] was used to transfer the coordinates of the receiver to the database.
The geographic locations of the tourist attractions were obtained using the UTM coordinate system and the Córrego Alegre horizontal datum plane to guarantee homogeneity during the field survey. According to the manufacturert the Garmin eTrex receiver has a mean localization error of $15 \mathrm{~m}$ (CEP_circular error probable) ${ }^{1}$ under normal conditions (i.e., using only the internal antenna) [10]. However, this study showed a mean error of $5 \mathrm{~m}$.

The coordinates of the surveyed points were entered into the database PostgreSQL/PostGIS using the free version of GPS TrackMaker software, which allows for the interface between the GPS and computer.

Thematic maps corresponding to "cultural tourist attractions", "natural tourist attractions”, "accessibility to tourist attractions", “accessibility to tourist attractions by private transportation" and "accessibility to tourist attractions by public transportation" were prepared from the data collected in the field regarding the presence of accessibility equipment such as reserved parking, ramps and bus stops (according to the program of inclusive accessibility). These maps were displayed in the GIS Web through the use of the software PostgreSQL/PostGIS, MapServer, and I3GEO.

\section{Development of the Web GIS}

The dynamics of OS software are the most interesting recent phenomenon in the computer field and have generated a level of interest similar to that stimulated upon the inception of the internet. More than 20 years of development have permitted advances to be made to better fit user needs. According to Stefanuto [11], this evolution is a result of a heterogeneous set of events, players and perspectives that are still finding their own paths but maintain the essence of an intense interplay between large active communities engaged with a common

\footnotetext{
${ }^{1}$ CEP (circular error probable): a circle's radius, centered at the true antenna position, which contains $50 \%$ of the points in the horizontal scatter plot.
} 
domain. This dynamic involves the development of software and related material, dissemination, support and encouragement for the use of OS software.

OS software has the same purposes as any proprietary software. In other words, it meets a specific need, such as running spreadsheets or editing images, among other uses [12]. Thus, what really makes OS software different from proprietary software is the type of license required. According to the open source software foundation, the OS license must ensure freedom to:

- execute the program for any purpose at any time at any point;

- study how the program works and adapt it to user needs (access to the source code is a prerequisite for this permission);

- redistribute copies;

- improve software and release improvements for the benefit of the whole community.

Therefore, OS software is about freedom rather than just being free. This freedom is defined primarily by the possibility of modifying, copying and freely using the software without restriction [11].

The OS concept is associated with a large social movement whose idea of freely using software is proclaimed to be the solution for the technological limits imposed by proprietary systems. According to Ref. [12], the speed of development of OS software has allowed great advances in information technology at costs compatible with benefits. As emphasized in the free guide of the Brazilian Federal Government [13], there are several reasons for the migration of public institutions to OS software:

- the need to adopt open standards for the e-Gov (electronic government);

- the higher level of security provided by OS software;

- the elimination of mandatory changes that proprietary systems impose on their users, with the threat of discontinued support for older versions;

- technological independence;
- the development of local knowledge;

- independence from a single supplier.

The rapid growth of OS software has saturated the market with various applications in several sectors. Thus, when one thinks about a geotechnology feature, the main concern is how to choose the best solution for a given situation. In this context, the choice of software used in this study was based on successful results reported in other studies using OS geotechnology. Thus, the framework used to develop this work is based on the integration of the Database Manager System PostgreSQL 8.3 with the PostGIS 1.3.5 module and the pgAdmin III database console, geographic information system quantum GIS 1.0.2, Apache HTTP server 2.2.4, MapServer 5.0.2 and application I3Geo4.0 for the development of the Web GIS interface. All of these are OS programs.

\subsection{Database Modeling Used in the GIS Web}

The abstraction of concepts and entities from the real world is an important part of GIS development. Its success depends on the quality of the implementation of real-world entities and their interactions within the database. In general, modeling seeks to systematize understanding about objects and phenomena to be represented in a computer system. Real-world objects and phenomena, however, are too complex to allow for a complete representation by the resources of the current DBMS. Thus, it is necessary to abstract objects and phenomena from the real world to obtain a simplified but convenient form of representation that is suitable for the purposes of database applications [14].

As previously mentioned, the authors chose for this study the PostgreSQL DBMS with an ORD (object-relational database), which incorporates some elements of object orientation while maintaining the relational structure.

Based on these concepts, a database named "acesso" was developed for Web GIS, which is linked to the entities "tipo_acessibilidade", "atrativo", 
“pontos”, “vagas_estacionamento" and “distancia_ponto”, as shown in Fig. 1. It is important to note that names in Portuguese language were given to the entities. These names reflect the nature of each entity.

The entities "tipo_acessibilidade” and “atrativo” are the most important of the "acesso" database. The first contains the accessibility codes, where code 1 indicates "accessible", code 2 indicates "partially accessible”, and code 3 indicates "inaccessible”. The entity "atrativo" contains information about tourist attractions such as sight name, address, type of attraction, and also the records "cod_acess", “acess_particular”, and “acess_publico”, which serve to classify the level of accessibility and type of accessibility to sights by private and public transportation.

Using the entity "atrativo", it was possible to make some geographic queries to build the "views" that formed the basis of thematic map visualization for the Web GIS.

\subsection{Preparation of Cartographic Base for Web GIS}

The cartographic database of Campinas used in this study was provided by EMDEC. Silva [15] highlights some points on the preparation of the base: "The work was initiated in 1991 with the digitalization of municipality maps by the municipalities computer associates S/A (IMA), using digitalization tables. Digitalization proceeded with non-standardized plants, using the available material: scales of 1:1.000 (center), 1:5.000 (periphery) and 1:10.000 (rural). Plants at a scale of 1:1.000 were provided by Paulista Power and Light Company (CPFL). It should be noted that these were derived from the expansion of plants at a scale of 1:5.000, which is not recommended. The layout prioritized undeveloped regions, with a higher rate of irregularities and, consequently, higher potential for tax collection. The planning secretary and the department of information and data registration cooperated by providing the layout and numbering of blocks. The secretary of public works collaborated with the catalogue of public sights.”

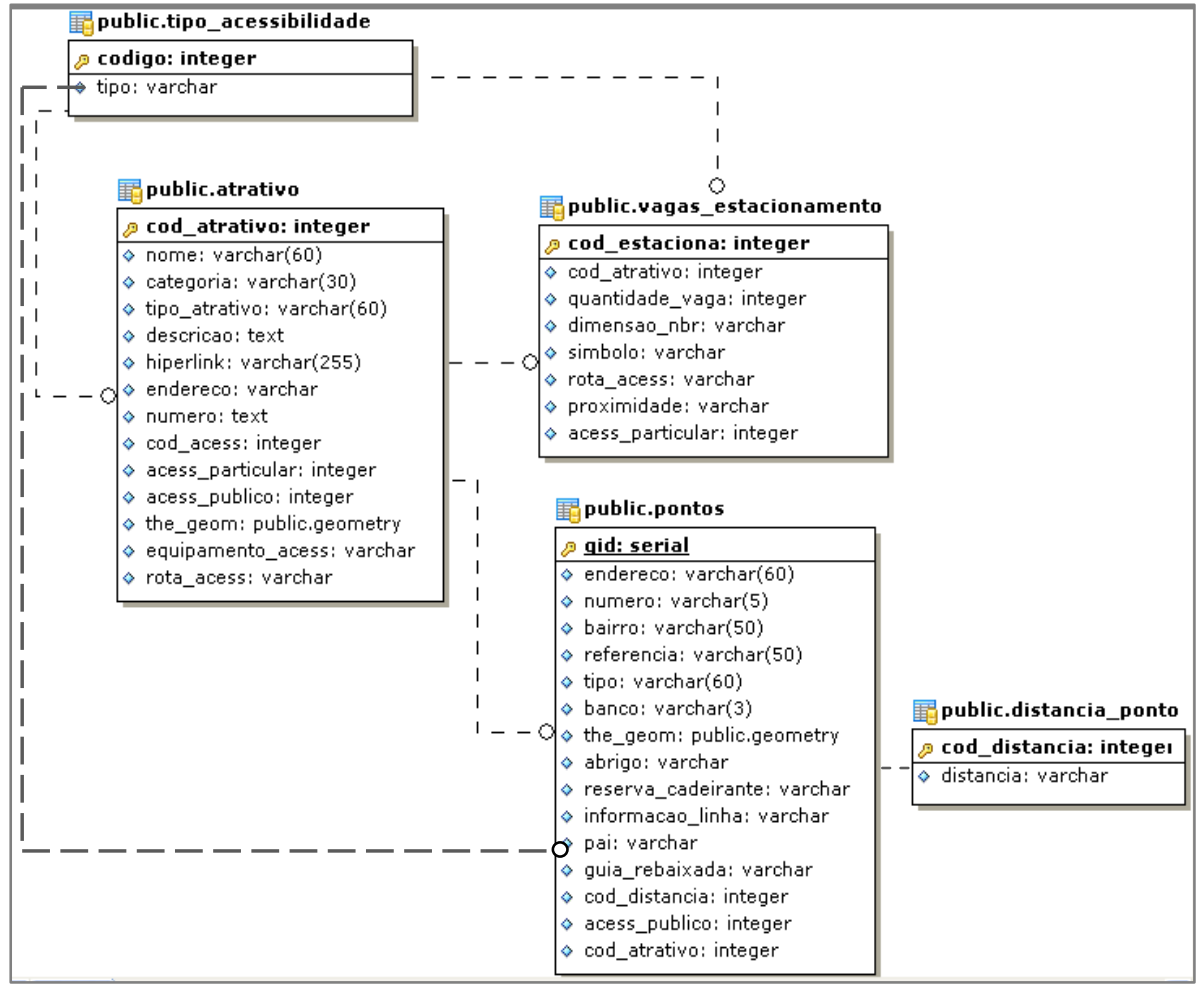

Fig. 1 ERM-entity-relationship model. 
It is noted that the base map was not produced by a continuous process of gathering and processing data; rather it was formed by a set of existing information obtained in different ways by several agencies.

The base was saved in the .dxf and .tab formats, which are compatible with the proprietary software Mapinfo used by EMDEC to analyze city bus routes. Seven layers were made available in the file formats above, using the Hayford ellipsoid, the Córrego Alegre horizontal datum and the UTM coordinate system as a reference surface.

A common false impression is that digital files are always ready for use. In fact, most of these files may have inconsistencies and thus require review before their inclusion in the GIS. It is noted that the files used for GIS are not just drawings but a representation of reality. Thus, when defining the shape of an object as a polygon, for example, it must comply with all the mathematical rules that define this geometry. The first step should be the preparation of digital vector files to verify the correct construction of the objects to be included in the GIS (point, line, polyline, polygon) as well as the datum and coordinate system used.

After assessing the layers provided by EMDEC in the AutoCad Map 2004 program, it was noted that they did not overlap properly. This occurred because the two layers were referenced on a geographic coordinate system unlike the other five, which were in the UTM coordinate system. In addition, the authors found that two layers had exactly the same data (both representing the blocks in the city of Campinas), but one was in line form and the other was in polygon form. The former was discarded.

To make the layers compatible with SGBD PostgreSQL, a series of programs were used to obtain pgsql files using the UTM coordinate system and the Córrego Alegre horizontal datum. GIS ArcMap, which is part of ESRI ArcGIR, with tool projections, which are part of the Arctool Box, was used to convert geographic coordinates to UTM.
To convert the files into the language used by the DBMS PostgreSQL-SQL, it was necessary to transform the files that were in the .dxf format to the .sql format. The OS software DXF2PostGIS was used for this conversion.

Because there was no open software available to transform .tab-formatted files directly to pgsql, .tab files were first converted to the .shapefile format, and these were converted to the .pgsql format. The conversion of the tab files to the shapefile format was performed using the FWTools tool, which is OS software available in Ref. [16]. The files were converted as follows:

ogr2ogr- [type of file to create] [name of file to create] [name of file to convert]

Fig. 2 shows the conversion of the tab file "sights" to .shapefile format.

The shp2pgsql was used to transform the .shapefile format into the .pgsql format. If the PostGIS spatial extension is enabled during PostgreSQL installation, then the sub-folder "bin" automatically appears and contains the executable file "shp2pgsql.exe". Using this tool, the files were converted as follows:

shp2pgsql.exe-s [SRID] [.shp file] [entity to be created in the database] $>$ [.sql file]

Below are some explanations of the command shown in Fig. 3:

- shp2pgsql.exe: PostGIS tool that enables the conversion of shapefile to pgsql;

- -s: parameter corresponding to the location in the southern hemisphere;

- 22523: together with the previous parameter, refers to the SRS ID (spatial reference system indentifier);

- Base.shp: name of the shapefile to be converted;

- Neighborhood: name of the entity that is created in the PostgreSQL database;

- Neighborhood.sql: name of the SQL file to be created.

The pgAdmin III database console was used to insert pgsql files into PostgreSQL. Thus, the sql files 


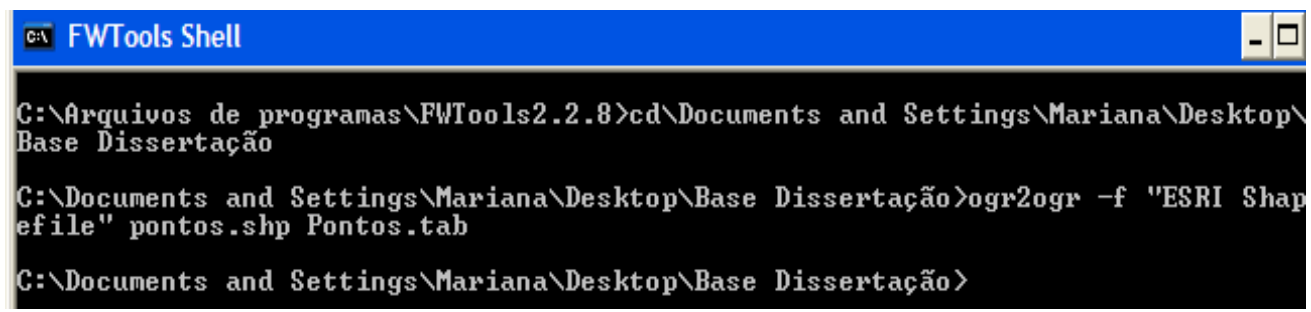

Fig. 2 FWTools command line.

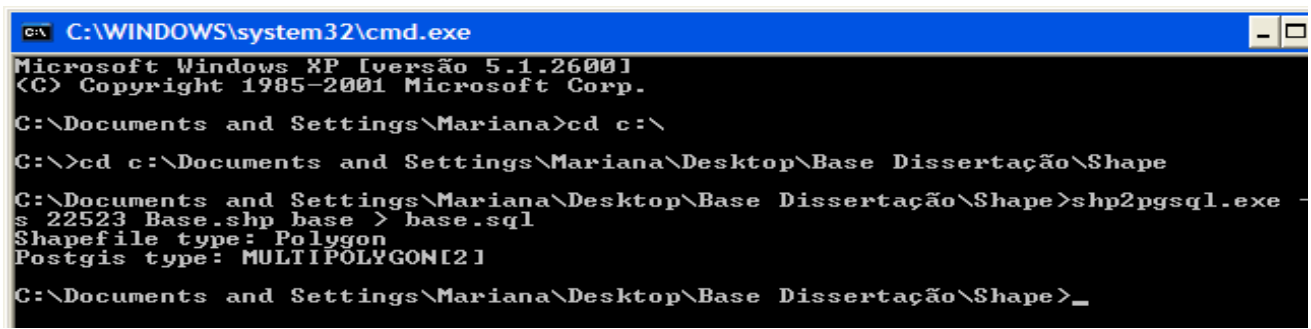

Fig. 3 DOS prompt screen with shp2pgsql command exeutionc.

were opened in pgAdmin III, and a query was executed, as shown in Fig. 4. When this procedure was complete, the layers of cartographic base of Campinas and other information received by EMDEC were integrated into a spatial database and could be introduced in the map file or imported by the quantum GIS data viewer.

The tool “Add Table (s) PostGIS” of the Quantum GIS software was used to visualize data inserted into the database. This tool allows access to the database created in PostgreSQL and the import of these tables to QGIS. Fig. 5 shows the tables imported from the database, enabling the visualization of blocks from the city of Campinas, city bus stops, terminals, city bus routes and other lines run by EMDEC.

Pictograms developed by Embratur in partnership with Denatran and Iphan were used to indentify tourist sites in the GIS. Once the pictograms were available on the website of Embratur [17] in matrix format (.tif files), it was necessary to convert them to a vector format so that they could be inserted into QGIS. The software VectorEye3 was used to make pictograms available in the .svg format.

\subsection{The Framework of Web GIS}

With goal of system efficiency and according to

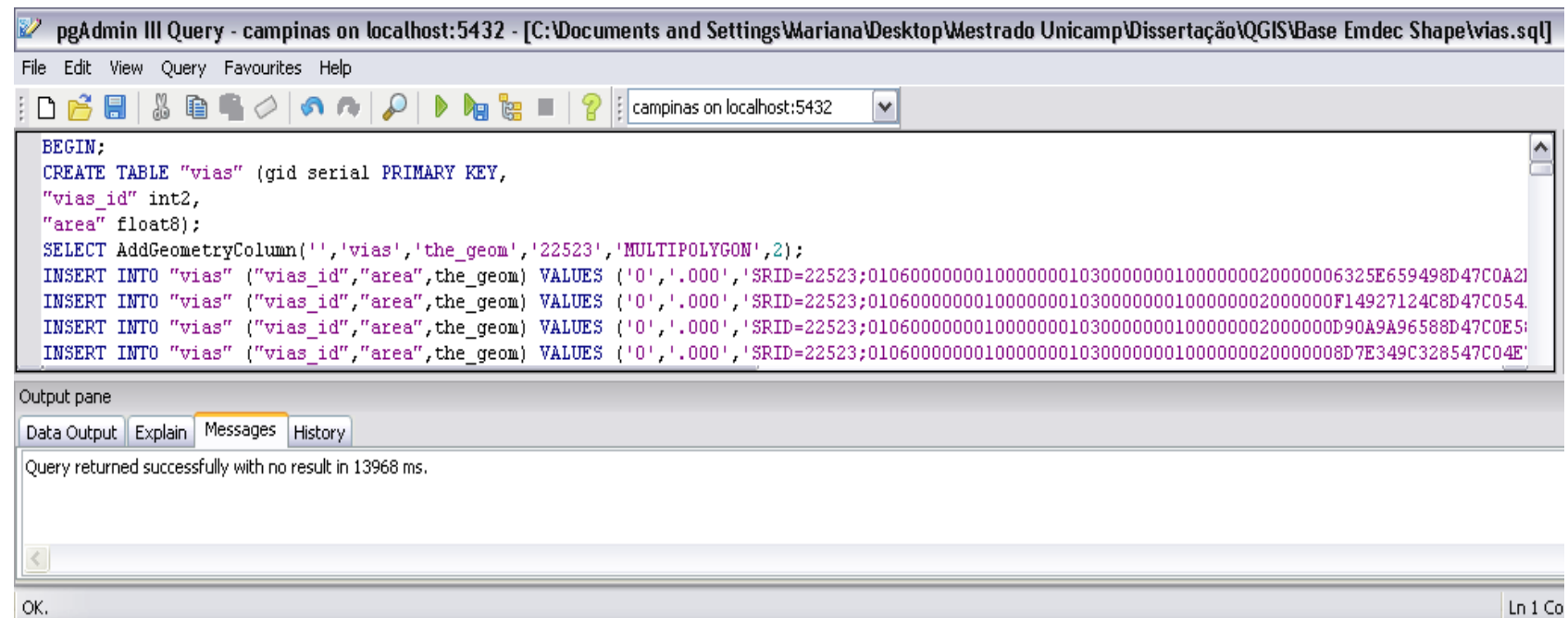

Fig. 4 PgAdmin III screen. 


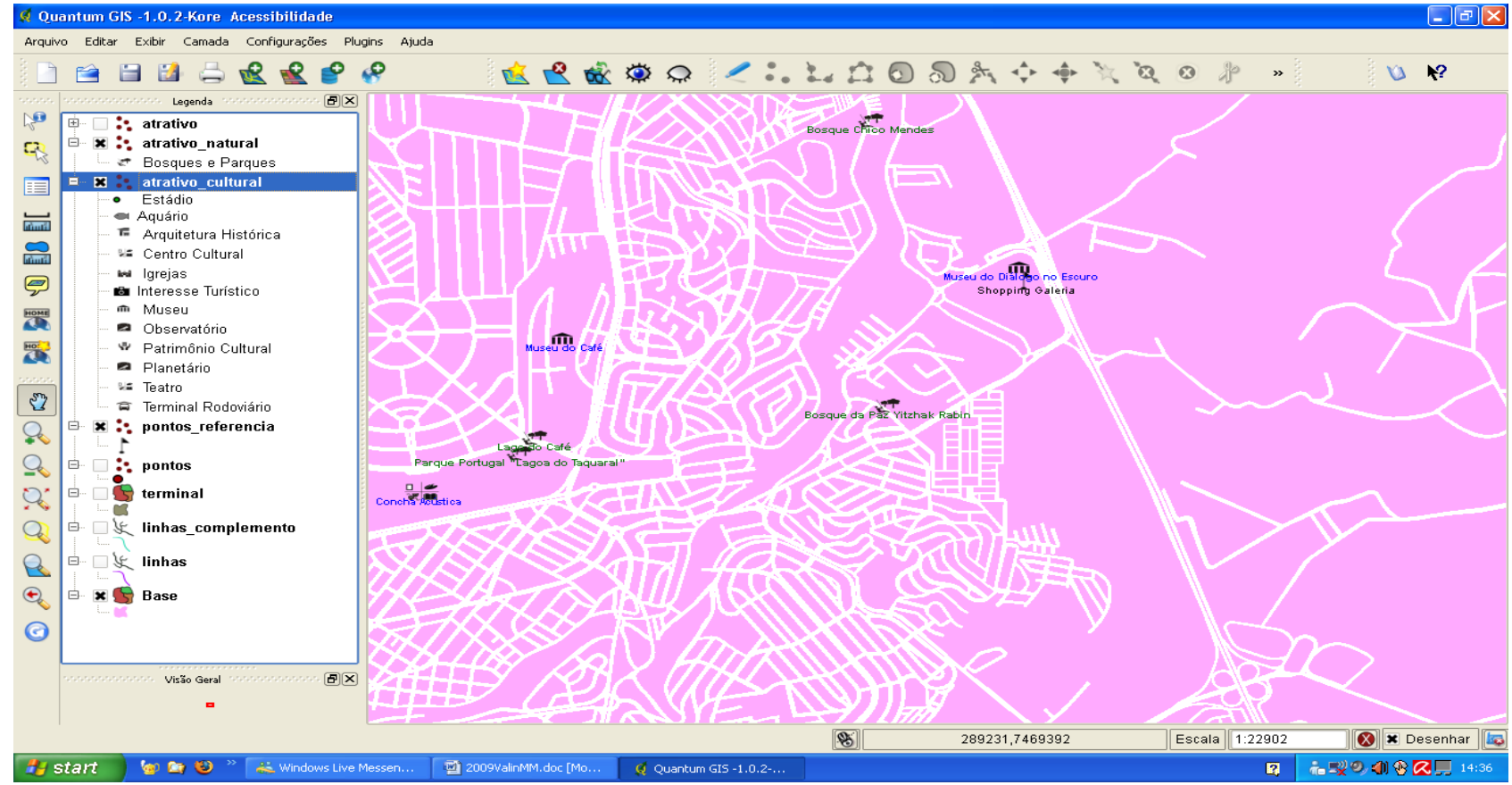

Fig. 5 Quantum GIS screen visualizing the data in the geographic database.

Paoli and Miscione [18], the development process was linked with user actions, emphasizing both technological and organizational aspects.

The client-server concept was applied to the Web GIS framework because it is both simple and efficient for building internet applications [19]. The major components of the framework are demonstrated for both the server and client side (Fig. 6). It should be noted that client-side communication is exemplified by the internet but can also be performed by any other network that supports TCP/IP. However, the client-server model was adopted by the internet because this technology is available to more people.

When the user accesses the project site [20] through his or her favorite browser, a request is sent to MapServer and received by Apache, which sends the files (codes of pages and images) to the client. The user then specifies the desired information by filling out form fields on the Web GIS pages (typing words of interest, selecting items, layers, etc.) [19]. For each change made to the information provided, a new request is sent to the server updating the map.

Data requested by the user can be retrieved in several ways. The simplest is through a direct query to the site, where the user can, for example, extract a picture, video, or a symbol. Other data are stored in a more complex structure, and in this case, the Web server forwards the request made by the user to I3Geo, which processes the request and returns the data to the Web server to be delivered to the user's browser.

\subsection{Installation and Configuration of I3Geo}

The I3Geo application was obtained free of charge from site of the Ministry of Environment [21]. It was used to develop maps for the Web interface. I3Geo was not developed for personal computers but for Linux web servers. Additionally, this software is not an executable file but a folder with multiple files and images. Therefore, this folder must be allocated at the root of a Web Linux server with the software necessary for proper functioning, such as the Apache Web server, PHP5 and PHP/mapScript, and MapServer with the cgi-mapserver package.

The folder "ms_tmp" was created in the same location as the I3Geo folder. The "ms_tmp" folder is used by Mapserver and I3geo to store temporary data such as map images. I3geo comes with a default view. To change it and insert the application developed in 


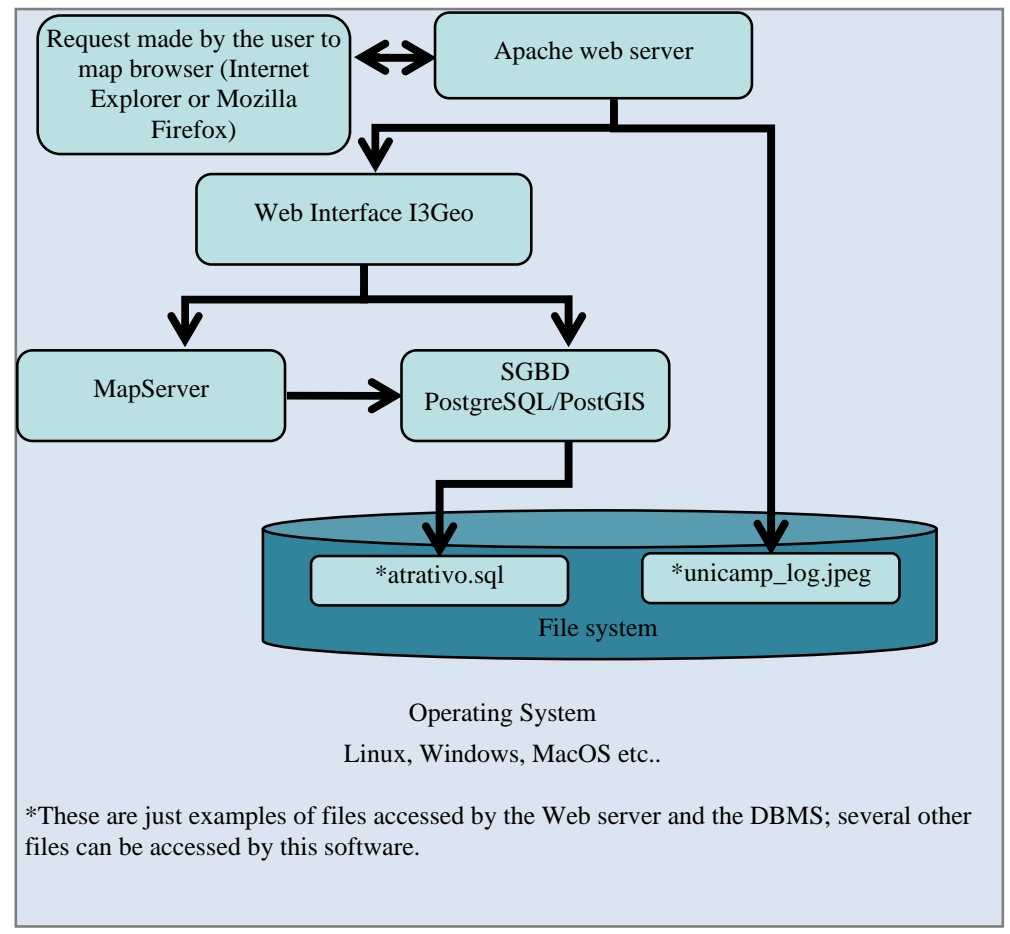

Fig. 6 The frame of Web GIS.

this study, it was necessary to modify the following files.

\subsection{1 ms_configura.php}

In this file found in “/var/www/html/i3geo/ms_configura.php”, there are variables responsible for the initial message of I3Geo:

\$mensagemInicia = "I3Geo version 4.0";

\$tituloInstituicao $=$ “.: accessibility to campinas tourist attractions.:";

The place where the folder ms_tmp was created was also indicated, as shown below:

\$dir_tmp = "/var/www/html/ms_tmp";

The location of the folder which the files .map had been stored was indicated, as well the place where the map file responsible for the map presentation in the initial screen of I3Geo was stored, as follows:

\$temasdir = "/var/www/html/i3geo/temas";

\$temasaplic = "/var/www/html/i3geo/aplicmap";

While configuring the ms_configura.php file, the location of the folder I3Geo was indicated in the Web Linux server:

\section{\$locaplic = "/var $/ \mathrm{www} / \mathrm{html} / \mathrm{i} 3 g e o " ;$}

It is noted that "/var/www/html" is the root level of the Web Linux server, where the folder "i3geo" was placed.

The link to the PotgreSQL/PostGIS database was also set. Use of this link is optional and is not related to data storage in I3Geo. However, because the application developed in this study is connected to the database, the variables were set as follows:

\$postgis_mapa $=$ "user $=$ mariana password $=\mathrm{xxxx}$

dbname $=$ acesso host $=$ localhost port $=5432 "$

\$postgis_con $=$ "user $=$ mariana password $=\mathrm{xxxx}$

dbname $=$ acesso host $=$ localhost port $=5432$ ".

3.4.2 geral1.map

The map file responsible for the map presented on the main screen of I3Geo is named geral1.map and is located at "var/www/html/i3geo/aplicmap/geral1.map". In addition to the definitions for project features such as map size, legend format, coordinate system, layer presentation, color, pictograms and font size, the pathways to directories containing fonts, symbols, shapefiles, images and temporary files are also stored in this file. The application settings used in this study are shown below:

FONTSET "/var/www/html/i3geo/symbols/fontes.txt” (path to the folder containing the fonts that are used by 
MapServer);

SYMBOLSET “/var/www/html/i3geo/symbols/sim bolos.sym" (path to the folder containing the symbols used on the map);

SHAPEPATH “/var/www/html/i3geo/aplicmap/ dados" (location of the shapefiles to be used by Mapserver);

IMAGE “/var/www/html/i3geo/temas/miniaturas/ minicampinas.png" (path to the folder containing the reference map);

IMAGEPATH “/var/www/html/ms_tmp/” (path to the folder where temporary files are generated);

IMAGEURL “/ms_tmp/" (URL path of the temporary folder);

TEMPLATE "/var/www/html/i3geo/aplicmap/ geral.htm" (path to the folder containing the template file).

I3geo is configured to use images in the png2 format by default, but this is not suitable for raster images. Because the pictograms used in this study were in the .png format, it was necessary to change the image format in the map file as follows: IMAGETYPE png1.

\subsection{3 simbolos.sym}

Map pictograms are stored in the file simbolos.sym, which is found at "var/www/html/i3geo/symbols/simbolos.sym". To configure this file, pictograms used in the maps were first added to the symbols folder in the .png format. Then the existence of these symbols was indicated by their inclusion in the simbolos.sym file, as described below:

Symbol
Name "arquitetura"
Type PIXMAP
Image "arquitetura.png"
END
Symbol
Name "futebol"
Type PIXMAP
Image "futebol.png"

\section{END}

\subsubsection{Database Connection}

To insert data from the field survey into the Web GIS, a PostGIS link was made in the map file geral1.ma. In addition, a geographic query was performed in the database to include layers in the Web GIS. The link and geographical query are as follows:

\section{CONNECTIONTYPE postgis}

CONNECTION "user $=$ mariana password $=\mathrm{xxxx}$ dbname $=$ acesso host $=$ localhost port $=5432$ "

DATA "the_geom from (select * from atrativo) as foo using srid = 22523 using unique cod_atrativo"

\subsection{Preparation and Presentation of Thematic Maps}

Based on collected data in the field, such as those regarding reserved parking, recessed pavements, and locations of bus stops for the program for inclusive accessibility, it was possible to prepare thematic maps that were then entered into the Web GIS via PostgreSQL/PostGIS, Apache, MapServer and I3Geo software. These maps were made available to users in the Web GIS format because the internet allows for the dissemination of information to a growing number of people and facilitate the use for the non-specialized public. Some techniques from the literature were considered in presenting information to end users because they make it easy to interpret cartographic maps.

Thematic data layers were used to map the accessibility of the tourist attractions of Campinas to avoid excess data. Thus, the Web GIS allows users to select the layers they want to view while keeping others hidden. Because there is much data to be jointly represented on the map base, the use of hidden data layers was fundamental to make each category understandable. Otherwise, it would be impossible to read and interpret all of the map themes simultaneously.

The data layers available on the Web GIS are "cultural tourist attractions", "natural tourist attractions", "accessibility of tourist attractions", 
“accessibility to tourist attractions by public transportation" and "accessibility to tourist attractions by private transportation”. The visualization of the Web GIS is shown in Fig. 7.

Pictograms standardized by Embratur (Brazilian Tourism Institute) in partnership with Denatran (National Traffic Department) and the Iphan (National Institute of Historical and Artistic Heritage) were used to reduce the polysemy of the first two superimposed layers. This proposal is feasible given that these symbols are common signs on tourist routes. Familiarity with these pictograms facilitates the association between the symbol and its meaning, thereby reducing the occurrence of dubious interpretations of signs shown on tourist maps.

It is noted that the symbols and their labels were displayed without overlap. Thus, when the scale of the map is small, some pictograms are hidden to preserve the readability of the map.

To indicate the levels of accessibility in the thematic maps “accessibility to tourist attractions”, "accessibility to tourist attractions by private transportation” and "accessibility to tourist attractions by public transportation”, green pictograms were used for accessible sights, yellow pictograms were used for partially accessible sights, and red pictograms were used for inaccessible sights. The pictograms used on the Web GIS and their meanings are described in Fig. 8.

The above proposal is based on the logic of Archela [22], which states that the tourist map should be unambiguous and logical so as to reveal the full content of the information provided and therefore direct the discourse of scientific work in a comprehensive, insightful, and critical way and socialize and demystify its content.

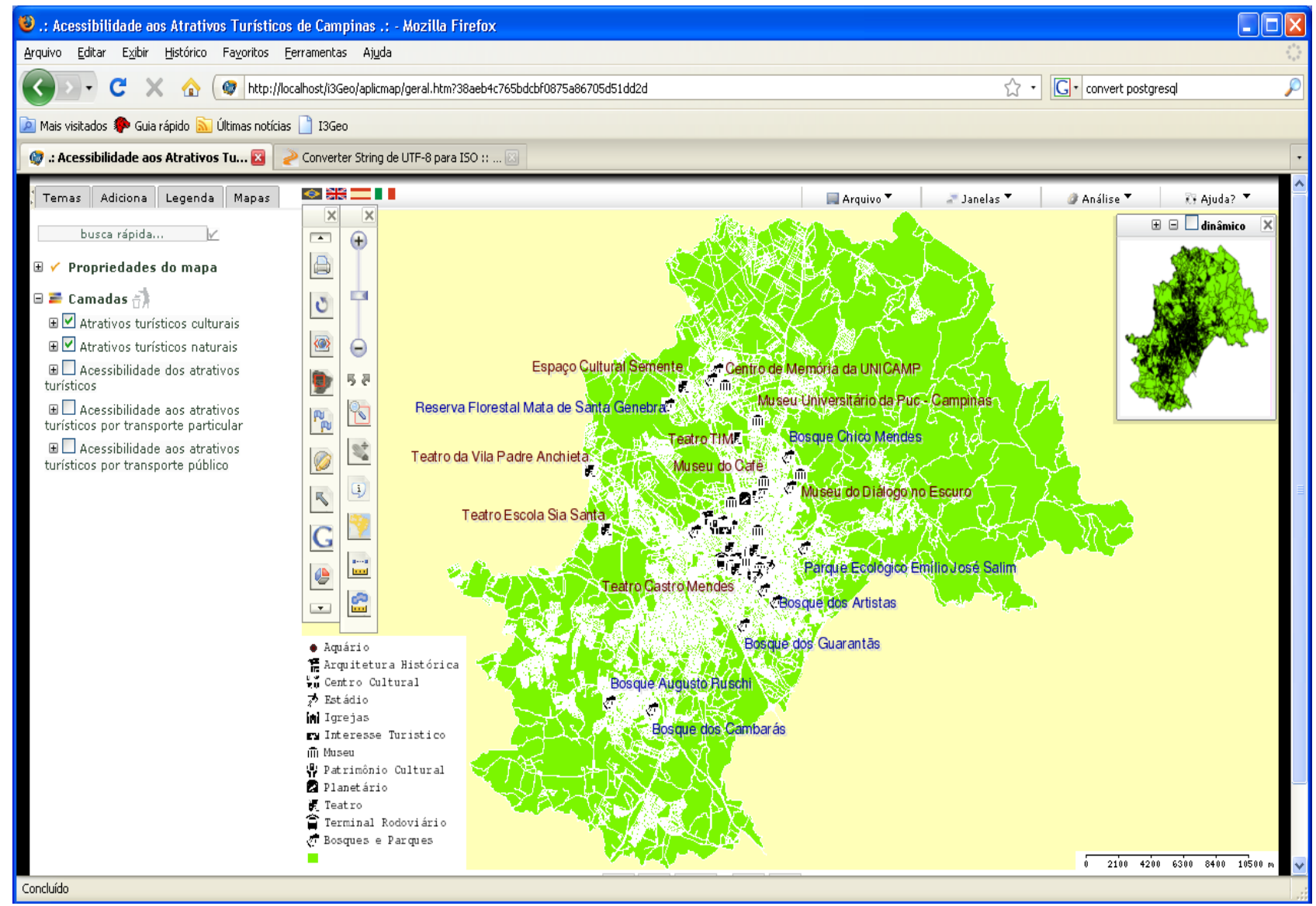

Fig. 7 Visualization of the Web GIS “accessibility of tourist attractions of Campinas”. 


\begin{tabular}{l|l}
\hline \\
\hline
\end{tabular}

Fig. 8 Pictograms used in Web GIS.

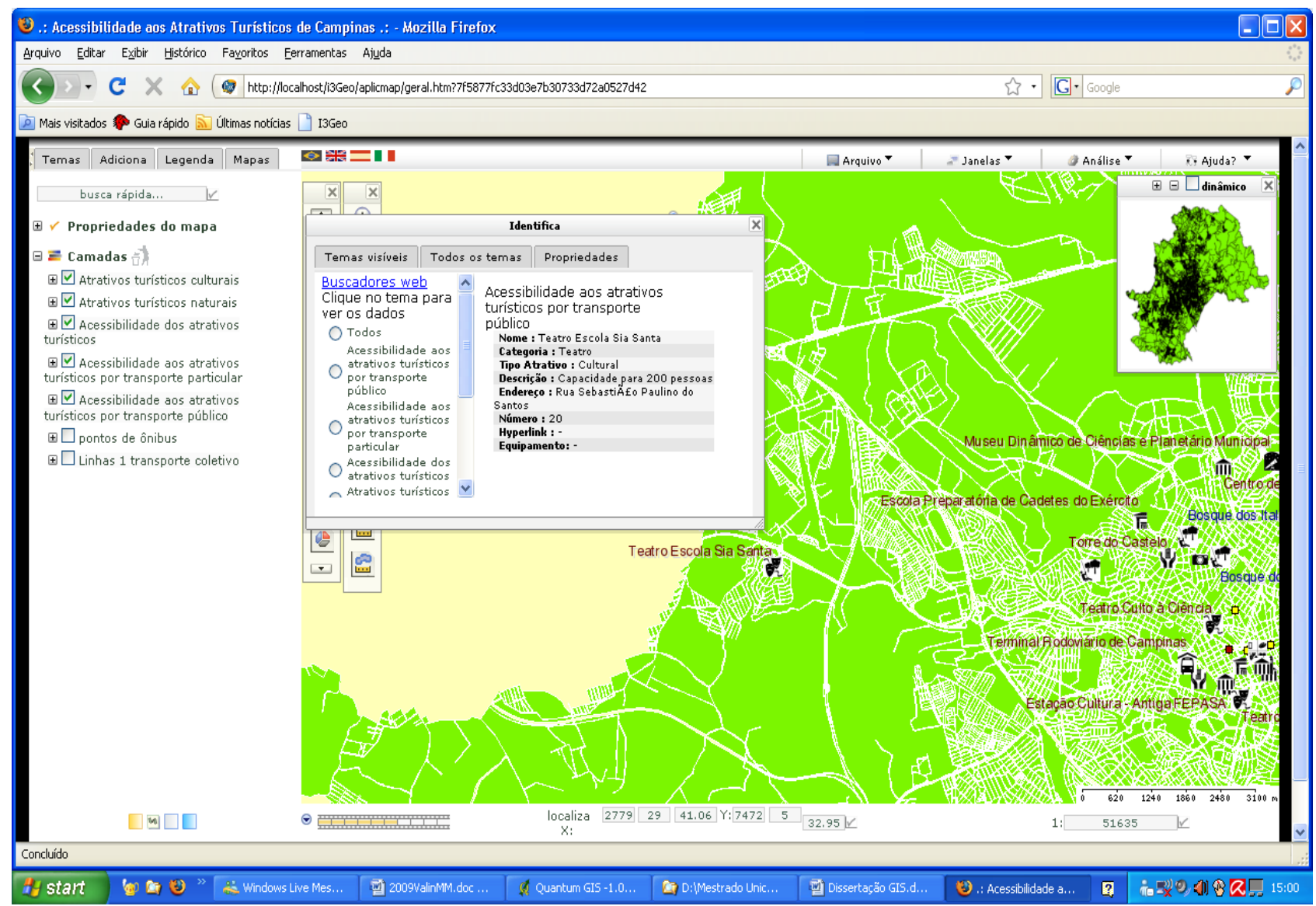

Fig. 9 View of the attributes of the Sia Santa School Theatre through the function "identify". 


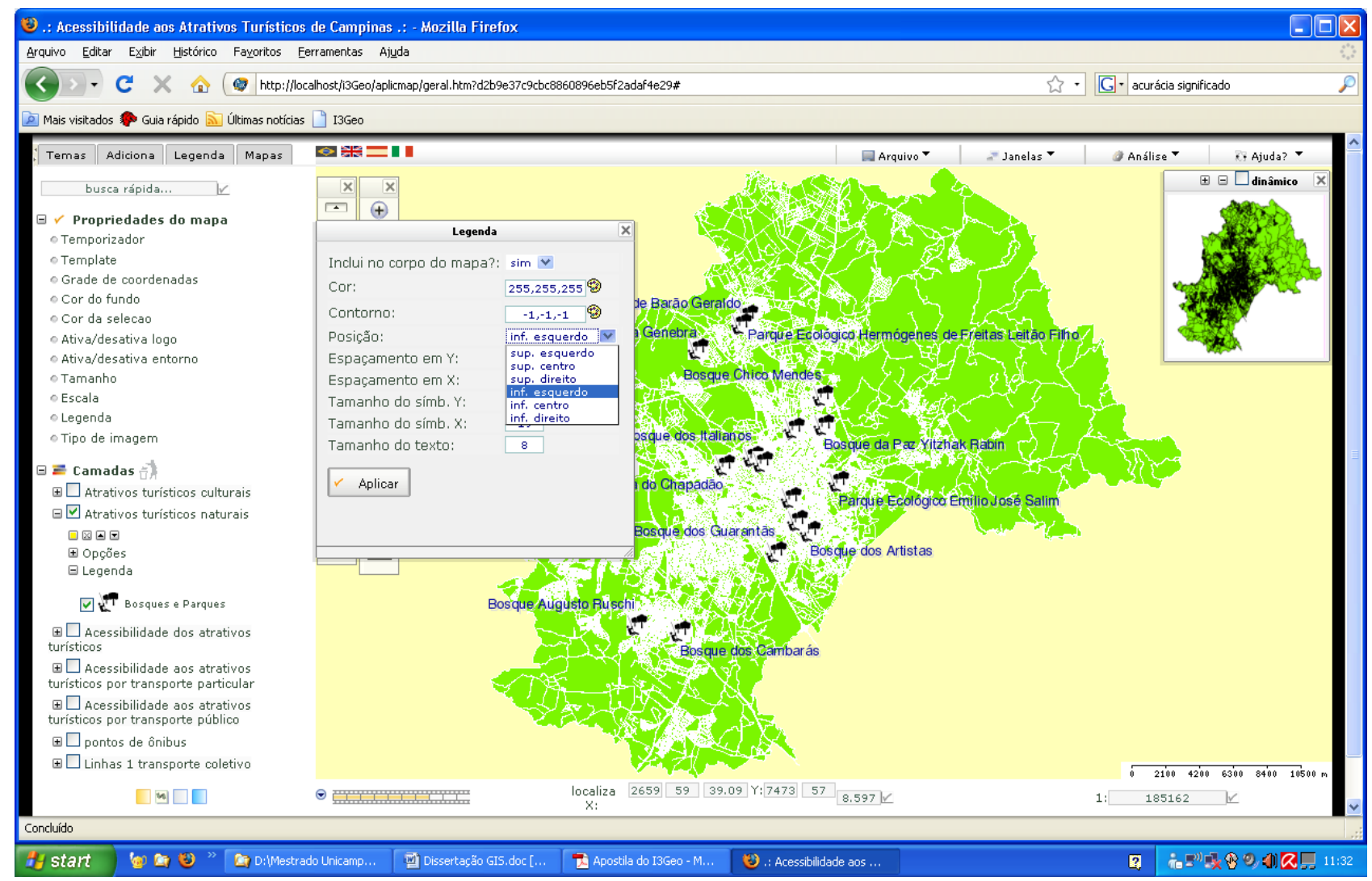

Fig. 10 Configuring the legend by the user.

The Web GIS developed in this study allows users to access information about a selected layer, such as the name of an attraction, addresses, the availability of accessibility equipment at the entrance of a tourist attraction and other information, as shown in Fig. 9. The tool also allows users to search for specific information with a "quick search" by using keywords and zoom-in and zoom-out functions and to determine the accessibility to tourist sites via private or public transportation.

The legend used to aid the interpretation of the thematic maps and their respective classes is interactive, floating, and can be hidden. In this way, the user can insert a legend in the map and place it in a convenient place. The legend can also be edited. Upon clicking it, a window opens with the available features (Fig. 10).

The Web GIS providing information regarding the accessibility of tourist attractions in Campinas is available in Ref. [20].
One innovation that could be considered in further studies is the transformation of the present Web GIS into Web GIS 2.0, which would integrate user-generated content, maintaining interoperability in terms of storage formats for geographic data and standards of communication between the database and GIS. This would create communication protocols that could be used in various systems [23].

\section{Conclusions}

The study showed that the contradictions are recurrent that, historically, are presented about several forms of exclusion manifested by prejudice, intolerance and segregation. In contrast to this, the tourism is presented as a way to promote social welfare as it is possible to provide the access to the touristic facilities, equipments, attractions and services, so that guaranteed equal rights and conditions to citizens are guaranteed.

As for the tourism, it is noted that nowadays there 
are no conditions of proper accesses. The technical visits to the local of touristic interesting urban areas of Campinas and District of Barão Geraldo, taking as references NBR 9050 (ABNT 2004), pointed out that despite significant improvements enforced by specific laws that guarantee to the disabilities or people with reduced mobility the right to citizenship, the physical environment of such places still has many obstacles that impede their mobility, showing its unreadiness to receive them. It was also observed that the actions on accessibility have been marked by the adoption of palliative solutions that hinder the optimal use of space and reinforce segregation.

Thus, it is essential to promote information about tourism and leisure facilities that offer access and enable its use by all citizens, regardless their difficulties or locomotion restrictions, hence this kind of information makes it easier to people with disabilities or reduced mobility in the different social and leisure activities, which contributes significantly for the social inclusion.

Presenting the levels of accessibility of the touristic attractions in Web GIS format, it is possible to the user require the adequacy of the environment in compliance with the current legislation, as well as allow the competent organs, always based upon universal design, to check the working systems and implement new equipments to provide accessibility in locals where they are not present.

Using the technologies surveyed, it was possible to develop and install the proposed system using OS software. This demonstrates that these solutions are at an advanced stage of development for geotechnology. Given its scalability, the OS software used in this study permits the dissemination of the spatial information to a large number of users who frequently access the internet.

The Web GIS developed in this study represents a great advance for the tourism industry because the data aiding tourists are concentrated in an interactive tool for visualization and query. Additionally, the broad coverage possible with the Web GIS shows the potential to generate a culture that benefits from adopting public transportation and sights, which fits the principles of universal design, thereby allowing more people to exercise their right to enjoy culture and leisure.

For the developed Web GIS presents information on the real world, which are subject to changes, it would be interesting to develop tool to help the update and maintenance of data. Thus, it will be possible to keep the completeness and consistence of the information presented.

In order to provide to the local government a tool of analyses, it is also recommended the creation of a tool inside GIS Web that allows the user to express his or her opinion about the accessibility of the touristic local, as well as about the facilities available for the means of transportation used (such as adapted parking spaces, proper ramps, easy access routes, etc.).

\section{Acknowledgments}

The authors thank CAPES (coordination for improvement of higher education personnel) for financial support through scholarship assistance.

\section{References}

[1] C.R. Duarte, R. Cohen, Developing a method for creating a guide accessible to Rio de Janeiro, in: Proceedings of 2nd International Seminar on Contemporary Visions, Rio de Janeiro, 2004, pp. 1-13. (in Portuguese)

[2] J.I. Miranda, Publishing Maps on the Web: Servlets, Applets or CGI? Embrapa (Brazilian Agricultural Research Corporation), http://www.cnptia.embrapa.br/ modules/tinycontent3/content/2003/doc28.pdf> (accessed Mar. 10, 2009).

[3] P.R. Ferreira, Method for generating maps of public transportation routes in an environment of open system for geographical information on the web, Master's Dissertation, Universidade Federal do Rio de Janeiro, Rio de Janeiro, 2007. (in Portuguese)

[4] H.G. Aragão, J. Campos, Web GIS builder: A visual tool for developing web GIS, in: Proceedings of 5th Latin-American Conference on Open Source, Foz do Iguaçú, 2008, pp. 1-6. (in Portuguese)

[5] V.A. Steinke, G.B.S. Silva, Spatial distribution of tourist 
services based on geographical information systems-GIS on the plano piloto of Brasilia (DF), Caminhos de Geografia 14 (16) (2005) 145-152. (in Portuguese)

[6] P.M.L. Menezes, M.C. Fernandes, Touristic cartography: New conceptions or old concepts and new conceptions, in: 21st Brasilian Meeting on Cartography, Belo Horizonte, 2003, pp. 1-8. (in Portuguese)

[7] Municipal Tourism Secretary Web Site, http://www.campinas.sp.gov.br/infotur/relatorio/index.ph p (accessed Mar. 10, 2009).

[8] NBR 9050, Accessibility to Buildings, Furniture, Equipment and Urban Spaces, Brazilian Association of Technical Standards, 2004. (in Portuguese)

[9] Trackmaker Trackers and GPS Solutions Web Site, www.gpstm.com (accessed Feb. 5, 2011).

[10] Garmin, What is WAAS? http://www8.garmin.com /aboutGPS/waas.html (accessed Feb. 5, 2011).

[11] G.N. Stefanuto, The Impact of Open Source Software on the Brazilian Software Industry, SOFTEX (association for promotion of Brazilian software excellence), Campinas, 2005. (in Portuguese)

[12] H.N. Uchôa, P.R. Ferreira, Geo-processing with Open Source Software: Version 1.0, Brazil, 2004, http://www.geolivre.org.br (accessed May 17, 2007). (in Portuguese)

[13] Open Source Guide: Reference for Migration to Open Source for the Federal Government: Version 0.9, Brazil, www.governoeletronico.gov.br (accessed May 13, 2008).

[14] M.A. Casanova, G. Camara, C. Davis, L. Vinhas, G.R.
Queiroz, Geographical Databases, Curitiba, 2005. (in Portuguese)

[15] D.L.N. Silva, Using a geographic information system to improve transportation specifically for people with physical impairments, Master's Dissertation, Universidade Estadual de Campinas, Campinas, 2004. (in Portuguese)

[16] MapTools Web Site, http://www.maptools.org (accessed Mar. 30, 2013).

[17] Embratur-Brazilian Tourism Institute, Brazilian Guide for Tourist Signs, 2005, http://www.embratur.gov.br /br/frame/index.asp?url=http://www.embratur.gov.br/hots itesinalizacao/conteudo/principal.html (accessed May 6, 2008). (in Portuguese)

[18] S. Paoli, G. Miscione, Relationality in geoIT software development: How data structures and organization perform together, Computers, Environment an Urban Sustems 35 (2011) 173-182.

[19] J.F. Kurose, K.W. Ross, Computer Networks and the Internet: A Top-Down Approach, Pearson Addison Edition Wesley, São Paulo, 2006, p. 576.

[20] Project Web Site, http://sig.fec.unicamp.br/i3geo (accessed Jan. 1, 2013).

[21] Ministry of Environment, http://mapas.mma.gov.br /download/arquivos/ (accessed Mar. 10, 2009).

[22] R.S. Archela, Image and graphical representation, Journal of Geography 8 (1) (1999) 5-11. (in Portuguese)

[23] B.D. Longueville, Community-based geoportals: The next generation? Concepts and methods for the geospatial Web 2.0, Computers, Environment an Urban Systems 34 (2010) 299-308. 